\title{
The Framing of Social Comparisons: Driving Upward and Downward Directions in Risky Decision-Making.
}

Marios Andrianos, Chrisanthi Nega, The American College of Greece - Deree.

\section{INTRODUCTION}

Contemporary research suggests that decision-making under uncertainty is sensitive to social cues and largely depended on behavioral influences.

\section{Prospect theory}

Proposes that risky decision-making is a dynamic process heavily influenced by subjective experience, and could be systematically explained by:

Framing Effects: Illustrate inconsistencies in decisionmaking when different framings of options influence the desirability of potential outcomes.

Loss-aversion: The tendency of people to be riskseeking facing losses as they ascribe greater value on losses compared to gains of equal size.

Probability Weighting Function: Describes the subjective evaluation of options, where its parameters are rooted in psychological predispositions.

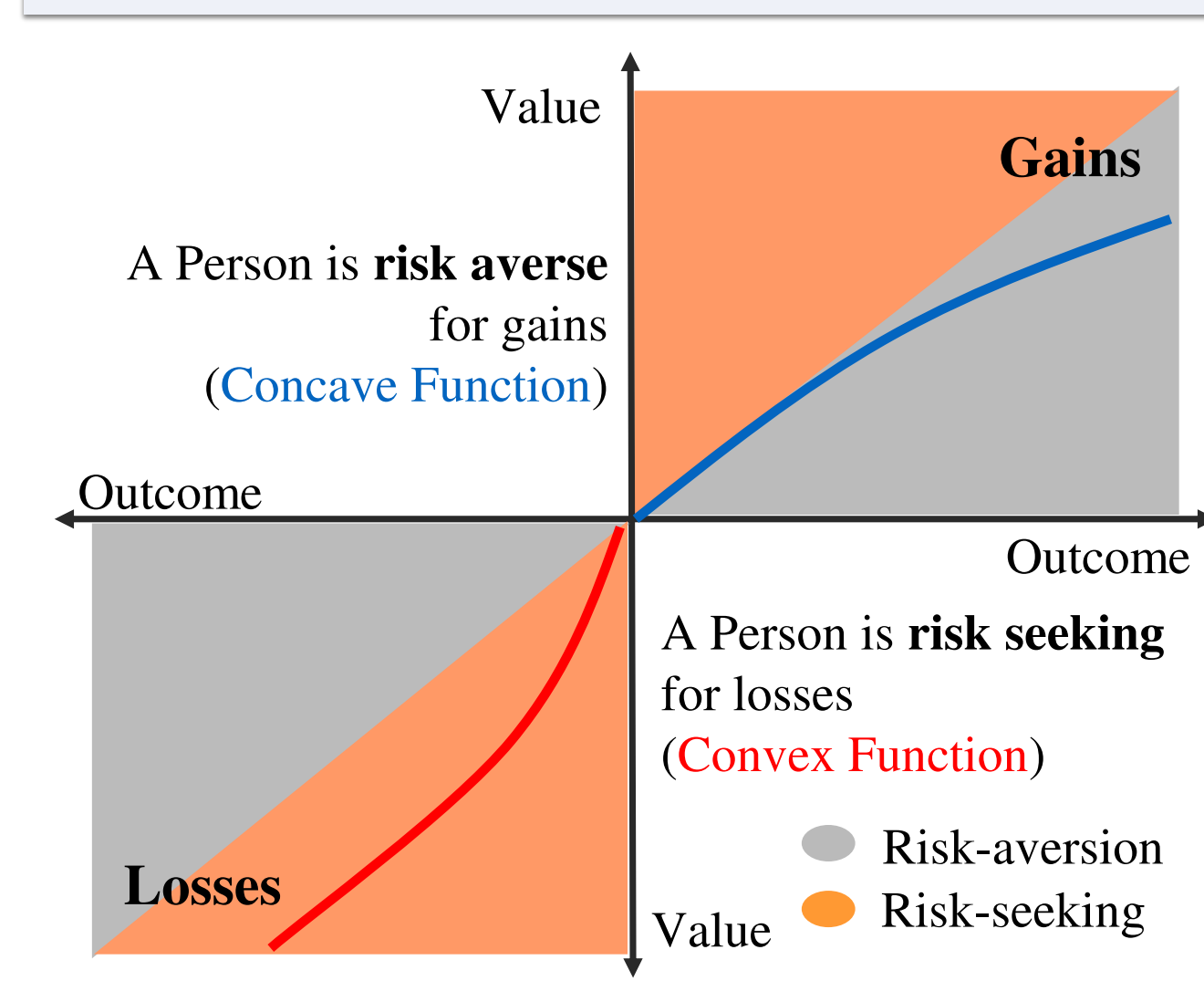

\section{Social Comparison Theory}

Humans are characterized by an "unidirectional drive upward" and strive to accomplish or preserve a superior relative status and alleviate discrepancies between their accomplishments and that of others.

Social comparisons and cues of relative competitive superiority or inferiority could influence risk-taking behavior. The distribution of wealth and social hierarchy have been conceptualized as crucial determinants. Frequently people adjust their decision to attain desirable outcomes and avoid unfavorable outcomes based on their relative social standings.

\section{Objectives}

Demonstrate how risky decision-making is sensitive to behavioral influences and social comparison cues addressed through attribute framing.

H1: Upward social comparisons would lead to a greater risk-seeking behavior compared to downward social comparisons.

H2: Individuals would be risk-seeking in the loss domain and risk-averse in the gain domain.

RQ1: How discrete absolute reference points reflect differences in risky decision-making?

RQ2: How different relative performance standings will affect individuals' risk-taking in gains and losses and their sensitivity to probability weighting function?

\section{METHOD}

Participants: 113 underoraduate students $(n=53$ males) with age range $18-27(M=22, S D=3.25)$

$$
\text { Design }
$$

$2 \times 2 \times 2 \times 3$ mixed factorial experimental design

IV1: Feedback for Ranking Position High rank vs. low rank

IV2: Framing of Social Comparisons Upward vs. downward

IV3: Domain of Risk Gain domain vs. loss domain

IV4: (EV) Expected Value Manipulation Equal vs. risk advantageous vs. risk disadvantageous

DV: Risky Decision-Making Overall scores and total sub-scores

\section{Materials \& Procedure}

Cognitive Reflection Test: 7 multiple-choice items, assessing cognitive impulsivity, as a test of problem-solving abilities.

Framing of Relative Performance Feedback: Purported relative performance feedback were presented in four different frames of social comparison in respect to the 4 experimental conditions.

Example: (High Rank - Downward Comparison) We have ranked the students based on their performance on the test you have completed that is an indicative of problem-solving ability. You performed better than the $90 \%$
Two-Outcomes Risky Decision-Making Task: Assessed risky decision making along with the sensitivity to distinguish and adapt to EV manipulation between certain and risky options. The task, including a gain and a loss domain, required subjects to choose between probabilistic options of potential win/loss of an amount of money.

\section{RESULTS}

A 4-way repeated measures ANOVA was performed to assess the main effects as well as the interaction effects of the four IVs on risky decision making (DV).

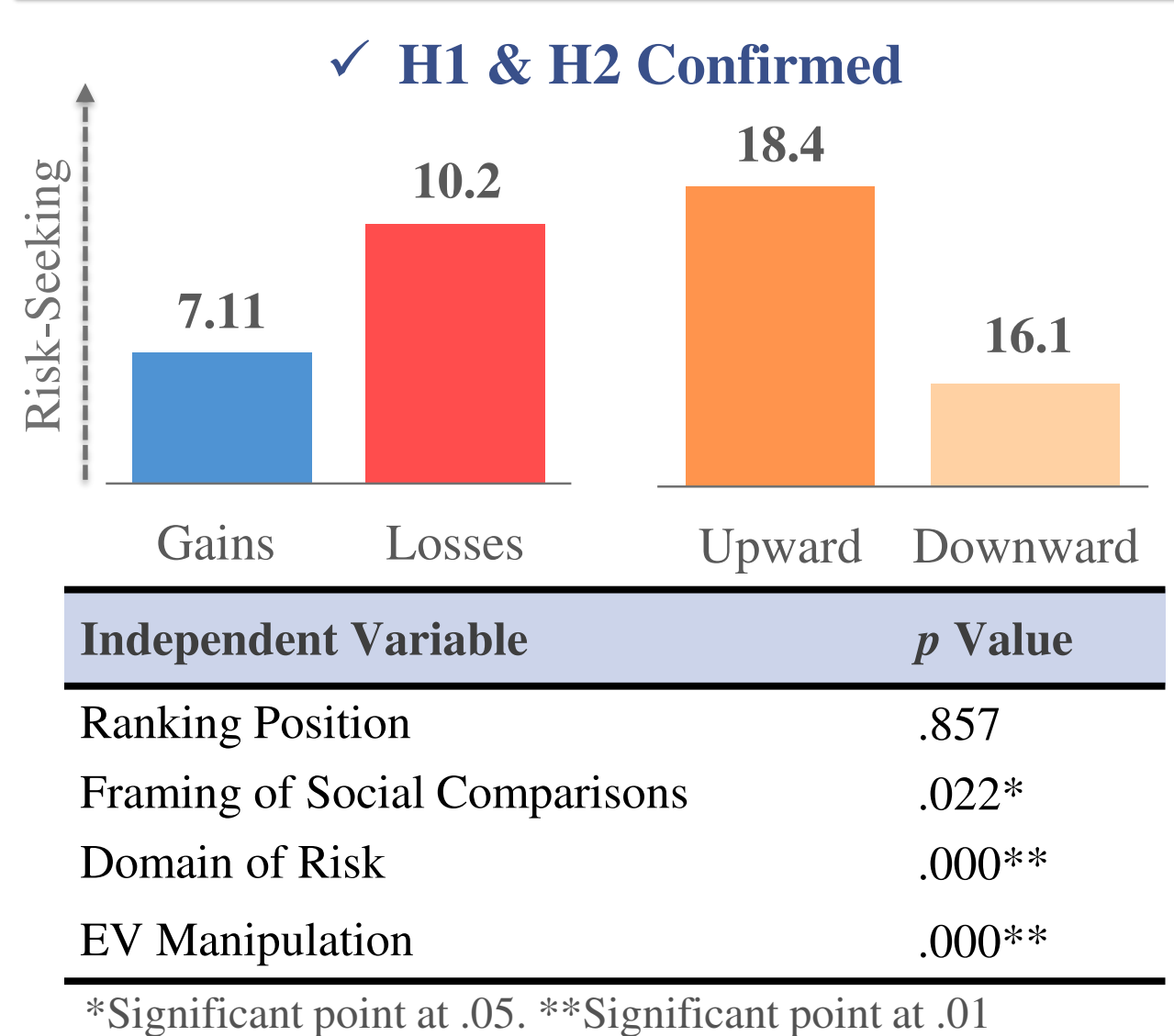

$\checkmark$ Rational Probability Weighting: Decisionmaking was sensitive to the EV manipulation between certain and risky options

- No main effect of IV1 on risky decision-making and no interactions effects of the IVs on the DV

Overall Risky Decision-Making Scores

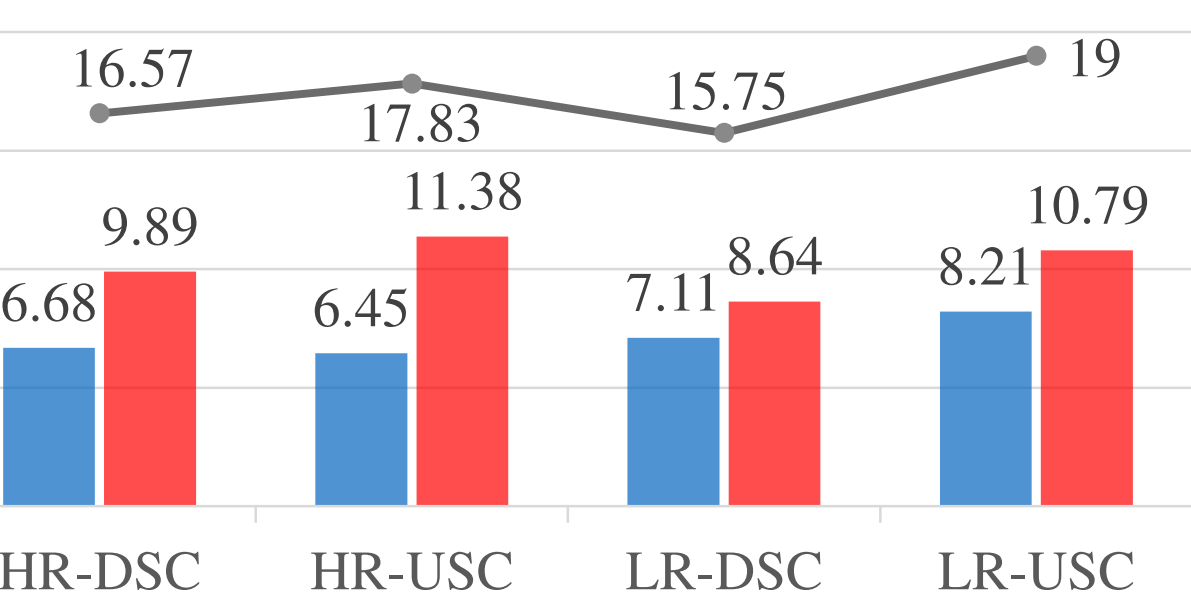

HR-DSC HR-USC LR-DSC LR-USC Gain Domain Loss Domain $\rightarrow$-Overall Scores Differences in risky decision-making between the four different relative performance feedbacks as product of IV1 \& IV2
Pairwise Comparisons of EV manipulation

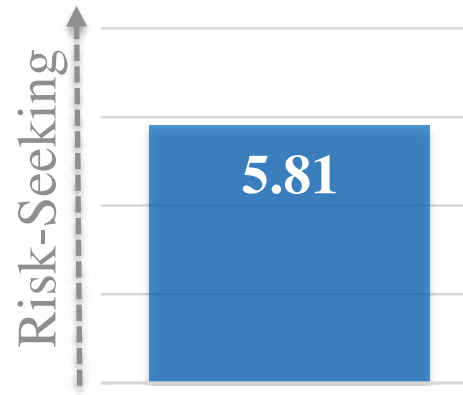

Equal

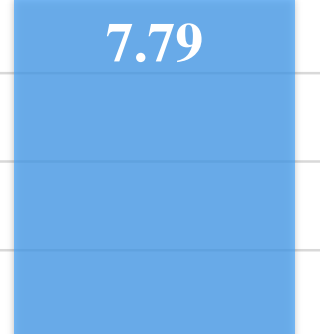

Risk

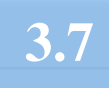

Risk
CONCLUSIONS

Decision-makers evaluate their utility not in terms of final wealth, but as a definite change in their current status-quo

- Risk-taking was not affected by ranking positions suggesting that relative performance matters more than absolute performance in social competition

Financial \& Social reference points are tantamount: cues of social inferiority lead in risk-prone economic behavior to compensate for the social loss Future Suggestions

Possible application of emotional and motivational dispositions in social cues of risky decision-making

> Assessments of behavioral influence in strategic decision-making through the context of social comparisons

\section{References}

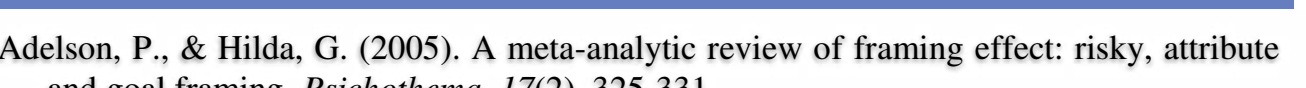
Gamba, A. Manzoni, E \& Stanca L (2016) Behavior. SSRN Electronic Journal. doi: 10.1007//1 1238-016-9562-z

one's achievents with of Business, And Psychology, 18(3), 301-321.

f., A Kahneman, D. (1992). Advaces in pospe

Chi-square of Goodness of Fit

Risk Seeking $\square$ Risk Averse

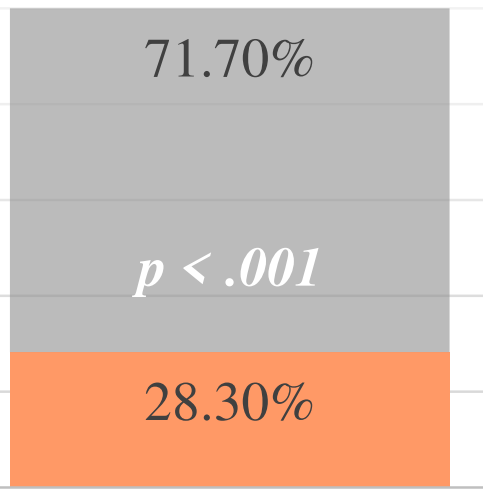

Gain Domain

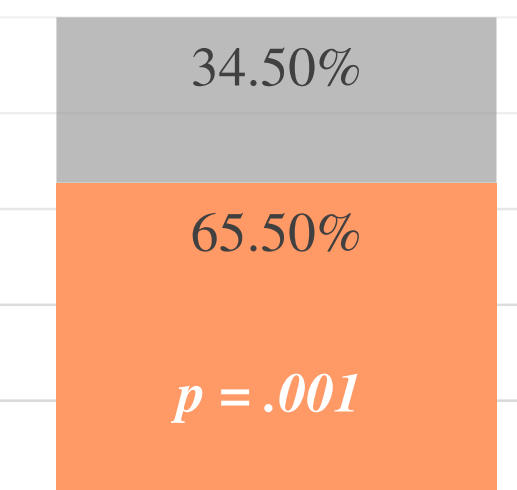

Loss Domain

Distribution of Risk-averse and Risk-seeking individuals in the Gain \& Loss domain 